\title{
ON USAGE OF VISUALIZATION TOOLS IN TEACHING MATHEMATICS AT UNIVERSITIES
}

\author{
Svetlana Asmuss ${ }^{1}$, Natalja Budkina ${ }^{2}$ \\ ${ }^{1}$ University of Latvia, Latvia; ${ }^{2}$ Riga Technical University, Latvia \\ svetlana.asmuss@lu.lv, natalja.budkina@rtu.lv
}

\begin{abstract}
The questions regarding the improvement of the learning system and the perfection of the quality are highly important in the work of teachers of mathematics at universities. One of the possibilities to make students interested to study mathematics is presenting the material by using its visualization. Visualization in teaching mathematics can be a powerful tool to explore mathematical problems and to imagine mathematical concepts and the relationship between them, to perform mathematical experiments and simulations. The paper is devoted to the authors' experience in using visualization techniques in teaching mathematics. There are many different software packages that can be used for creating illustrations or/and animations for problems from different study courses. The talk deals with such special tools as GeoGebra, Mathematical Visualization Toolkit (MVT), Microsoft Project, RStudio, Java Modelling Tools (JVT). By discussing some specific examples of problems from Mathematical Analysis, Methods of Optimization, Operations Research, Numerical Methods, Probability Theory and Mathematical Statistics, the authors show how the considered tools could be effectively used for illustration of theoretical results or solving problems during the practical works. The possibility to use the mentioned tools for the analysis of real-world systems working out study projects and bachelor's theses is also discussed.
\end{abstract}

Keywords: teaching mathematics, visualization, animation, software packages.

\section{Introduction}

The problems concerning the improvement of the learning system and the perfection of the quality are of paramount importance in the work of teachers of mathematics at universities. Unfortunately, it is necessary to point out the decline in the knowledge of mathematics of schoolleavers, low motivation of students and their poor ability to learn systematically. One of the possible methods to make students interested and motivated to study mathematics is presenting the material by using the possibilities of different computer technologies. Many teachers now use the tools like Power Point, web applications, applets, visual material in their lectures. The importance of visualization can hardly be overestimated in general cognitive skill acquisition and problem solving processes. Modern students, who have got accustomed to bright images from the early childhood, perceive the material with elements of visual representations much better. The purpose of using graphs, diagrams, charts, animations is to make some parts of the study material easier to understand, more specific and descriptive. Visualization allows to reduce complexity when dealing with a multitude of information. In combination with modern numerical methods, it allows to perform mathematical experiments and simulations, to construct the models of different real-world systems and to analyse the work of them.

The role of visualization in teaching mathematics has been the subject of much research (see, e.g. [1-4] and references therein). Many reports, devoted to the role of visualization, to using the visualization tools or difficulties with them in learning and teaching mathematics were presented during the Annual Conferences of the International Group for the Psychology of Mathematics Education (review of some of these presentations from 1988 till 2005 could be found in [5]). Regardless of their focus, these papers nearly all show that success in learning mathematics owes

among other things to visualization skills.

Zimmermann and Cunningham wrote already in 1991: "Computing technology is making it much more rewarding for mathematics to use graphics, and in turn mathematics is showing an increased interest in visual approaches to both teaching and research" ([6]). During this time a lot of different papers about the role of the computer in teaching mathematics have appeared. For example, the focus of [7] is the use of digital technologies in mathematics teaching and learning in countries across the world, such themes are mathematics and mathematical practices; learning and assessing mathematics with and through digital technologies; teachers and teaching; design of learning environments and curricula; implementation of curricula and classroom practice. The article [8] reviews some studies on visualization in learning mathematics, describes some principles both for information design and for user experience, and discusses their relevance in creating effective interactive visualization in learning 
mathematics. Many reports were devoted to the same themes during the conference "Technology and Psychology for Mathematics Education" in March 2019. In this article, for the sake of brevity, we do not discuss the importance of visualization in the teaching process, but limit ourselves mainly to our experience in using some software packages for visualization in teaching mathematics. A list of the topics of mathematics, where the visualization technique can be used as an additional effective tool for improving the quality of lectures and practical works, is presented in the next paragraphs. All considered examples from these topics are shown with comments on possibilities of using different software packages for visualization of solutions of these examples or illustration of the mentioned mathematical concepts. Effectively usage of the visualization technique in modelling and analysis of real-world systems or in scientific research is shown by examples of bachelor's and master's theses in the last paragraph of the paper.

\section{Materials and methods}

Different topics of mathematics, where software packages could be used for visualization of solution of problems, understanding mathematical concepts, illustration ideas and influence of quantities or parameters in different topics, are considered in this paragraph. Shown examples are probated by the authors of the paper in their practice.

\section{Linear and Quadratic Programming}

Let us start to consider examples with the optimization problems, which, on the one hand, appear in many courses (e. g., methods of optimization, operation research, game theory, sampling survey, mathematical analysis), but on the other hand, their solving could be very easily demonstrated geometrically by using Geogebra.

GeoGebra is the free software with interactive geometry, algebra, statistics and calculus applications intended for learning and teaching mathematics On the one hand, GeoGebra is an interactive geometry system. You can do constructions with points, vectors, segments, lines, polygons and conic sections as well as functions while changing them dynamically afterwards. On the other hand, equations and coordinates can be entered directly. It finds derivatives and integrals of functions and offers commands [9].

We can suggest GeoGebra for creating a set of examples for the linear programming problem and dual problem, for quadratic programming, for nonlinear programming, for solution of some problems from Game Theory, for sequences of functions, for series of functions, for Riemann sums for integrals, for interpolating and smoothing splines, for Lagrange interpolating polynomials and so on. In the paper we consider some of these examples in a more detailed way. The visualization in all of them is based on using the slider, which changes its value simultaneously with geometrical changing in the picture.

For geometrical illustration of a linear programming problem (in the case of two variables) by GeoGebra (see Figure 1), it is necessary to constrain the feasible region and to find solutions by moving the straight line, which corresponds to the objective function level. The value of the objective function changes on the screen of GeoGebra as value of the slider according to the location of the objective function level line.

Example 1. To maximize $f(x, y)=2 x+3 y$ under constraints $x+y \leq 6, x+2 y \leq 8, y \leq x+2, x, y \geq 0$.

The solution of this problem is $(4 ; 2)$ with the value of the objective function at this point equal to 14. It corresponds to the vertex $\mathrm{C}$ on the image of geometrical solution of this problem by GeoGebra (see Fig.1).

Geometrically it is easy to show how the solution gets different values by changing the coefficients of the objective function.

By analogy with the problems of linear programming, the problem of quadratic programming could be considered. 


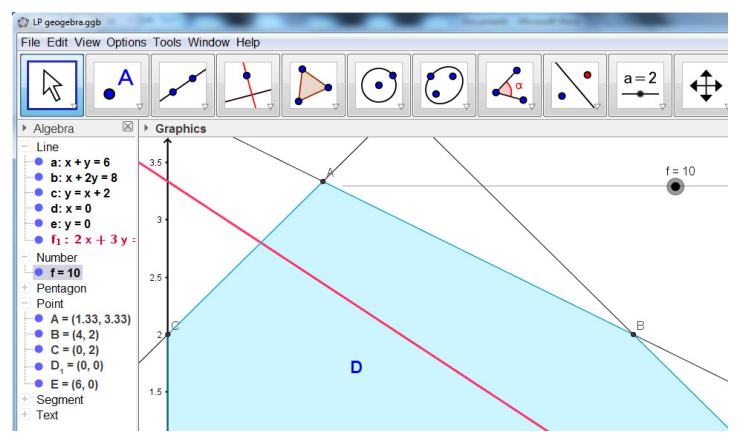

\section{Fig. 1. Geometrical illustration of solving Example 1 via GeoGebra (step with value of objective function 10)}

\section{Sequences and Series of Functions}

The next example demonstrates using of GeoGebra in the solution of problems on convergence of sequences of functions. Suppose $\left\{f_{n}\right\}$ is a sequence of functions sharing the same domain and codomain. The sequence $\left\{f_{n}\right\}$ converges pointwise to $f$, if and only if $\lim _{n \rightarrow \infty} f_{n}(x)=f(x)$ for every $x$ in the domain. By using GeoGebra, the notion of pointwise convergence becomes clearer. In order to show it, let us consider the following example.

Example 2. The sequence $f_{n}(x)=\left(1+\frac{x}{n}\right)^{n}$ convergences to the function $f(x)=e^{x}$.

The changes of the parameter $n$ are shown by slider. By increasing $n$, the graph of the $n$-th term of sequence becomes closer to the graph of limit and since some value of $n$ they visually jump together (see Figure 2).

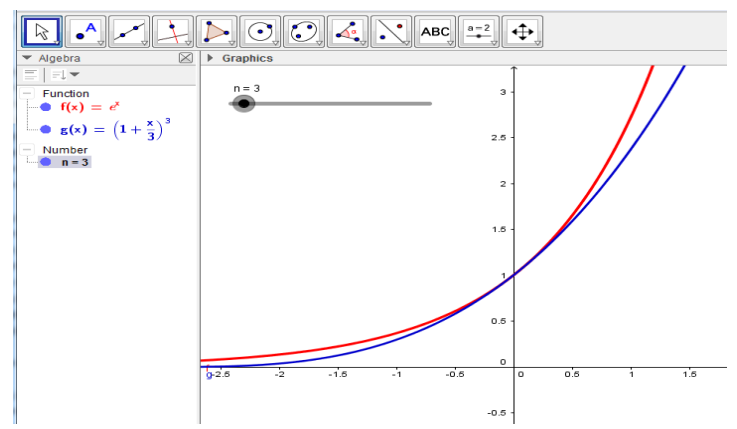

Fig. 2. Graph of $f(x)=e^{x}$ and graph of the third term of sequence from Example 2

The demonstration of the last example is not difficult by GeoGebra, because the number $n$ is in the formula of $n$-th term, but the situation is quite different in the case when the formula of the term absolutely changes, depending on the values of $n$, or the formula of $n$-th term is obtained by using the formula of the previous term (as, for example, in the formula of partial sums of series). In that case we offer the method, by which the $n$-th term appears with disappearing of the previous $(n-1)$-st term for the sequences or the graph of partial sum changes, when the next term of the series is added. The main idea of the method is to consider the changes of the values as the values of discrete function. For it we use function " $\max [x, 0]$ ", which gives the biggest value between 0 and the value of $x$.

Let us consider the case of series as the example of using such method. The dynamic visualization for description of a function by, for example, Taylor series, is more useful for students than the static image of graphs of some partial sums. The additional descriptions and explanations about accuracy of approximation are necessary for the static picture. The dynamic visualization itself shows how this accuracy changes by increasing the number of the terms in the partial sum.

$$
f(x)=f\left(x_{0}\right)+f^{\prime}\left(x_{0}\right)\left(x-x_{0}\right)+\frac{f^{\prime \prime}\left(x_{0}\right)}{2 !}\left(x-x_{0}\right)^{2}+\ldots+\frac{f^{(n)}\left(x_{0}\right)}{n !}\left(x-x_{0}\right)^{n}+R_{n}(x) .
$$


It is not so easy to construct this visualization by GeoGebra. The following formula for description of the partial sums of Taylor series is suggested by the authors.

$$
\left.S_{n}(x)=u_{1}(x)+\max [n-1,0] u_{2}(x)+\max [n-2,0]\left(u_{3}(x)-u_{2}(x)\right)+\max [n-3,0]\left(u_{4}(x)-u_{3}(x)\right)\right)+\ldots
$$

where $\quad u_{n}(x)-n$-th term of the series. According to this formula by increasing $n$ by one ( $n$ is natural number), the additional term appears in the partial sum.

Example 3. $\sin x=x-\frac{x^{3}}{3 !}+\frac{x^{5}}{5 !}-\cdots+(-1)^{n} \frac{x^{2 n+1}}{(2 n+1) !}+\cdots$

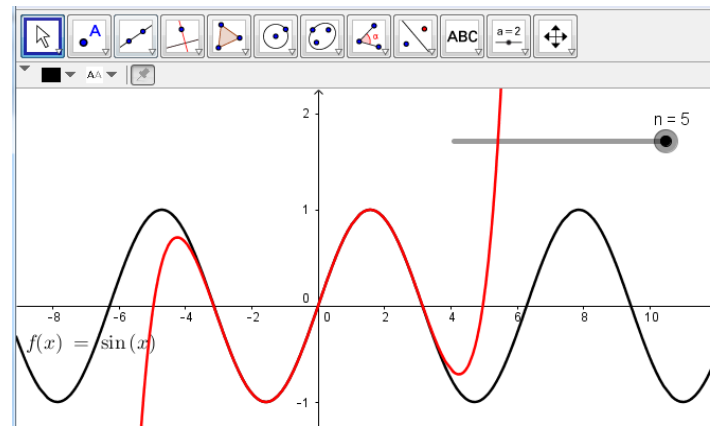

Fig. 3. Graphs of $f(x)=\sin x$ and of the fifth terms of Taylor series via GeoGebra

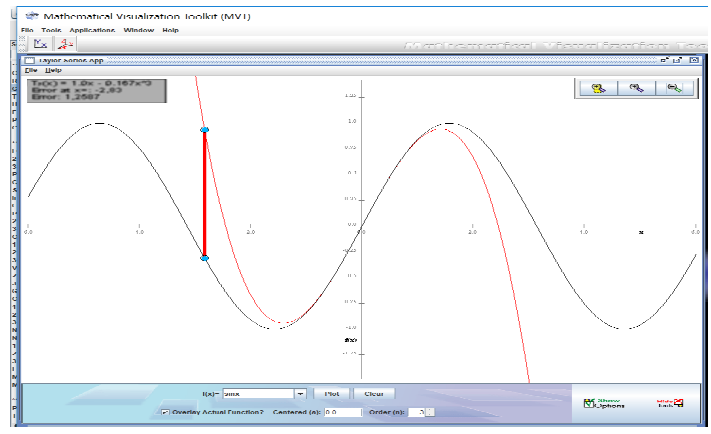

Fig. 4. Taylor series of order 3 for function $f(x)=\sin x$ in MVT

The other software also could be used for such type of example. It is Mathematical Visualization Toolkit (MVT). MVT is a set of visual and computational tools designed to help students better visualize the concepts of Calculus. It contains Scientific calculator, Plotting tools, Numerical tools, Linear algebra tools, Differential equations tools, Content-specific applications, Other Calculus visualization tools, Tutorial-style help system ([10]).

In the opinion of the authors of the paper this tool mainly could help generate pictures that would be tedious or impossible to generate by hand. It could be used, for example, for constructions of graphs of functions (including in polar coordinates), curves and surfaces (including in cylindrical and spherical coordinates), ODE vector field, etc. MVT visualizations can be used also for explanation and easily understanding of some concepts, for example, for Riemann sums for integrals, for Taylor series, for Lagrange interpolating polynomials, for interpolating cubic splines and so on.

Let us return to the example of Taylor series. Taylor Series application of MVT shows the Taylor polynomial of order $n$ generated by $f$ at $x=a$ (see Figure 4). At the bottom of the Taylor Series Window is an input region. This is where we specify the function of which we wish to view the Taylor Polynomial $(f(x)=)$, its domain and the order of Taylor Polynomial $(\operatorname{Order}(n))$. The Taylor Series for the function appears in the "answers" field in the upper left of the graph. The difference between the values of the function and Taylor Series also appears in this field (Fig. 4, at $x=-2.83$ error $=1.2587$ ). The possibility to find this difference is not presented in GeoGebra.

Analogous examples could be created by GeoGebra and MTV in the case of Riemann sums for integrals. 
Taking into account that the examples considered above could be visualized by both GeoGebra and MTV, in conclusion of this paragraph we give our subjective brief comparative analysis of both tools.

- Solver: The possibilities of GeoGebra are more powerful. For some themes MTV as solver could not be considered, e. g., it is not possible to see analytical solution of differential equations, formula of derivative, only its graphical view. But MTV gives a possibility to consider the solution of problems by approximate methods (solution of equations, integrals and so on), solve the interpolation problem by Lagrange polynomials, cubic splines. We note that for applications with approximation MTV, in difference from GeoGebra, it gives the possibility at once to see the difference between values at point of a given function and constructed approximant (as it is in Example 3, Fig. 4).

- Possibilities of construction of graphs are widely presented in both tools, experienced user has no problem to construct any graph, line or surface by these tools, including it in specific coordinate systems. GeoGebra in difference from MTV gives the possibility to correct fonts of labels and thickness of lines, which is important in the case of using graphs for presentation.

- Animation: MTV has minimal possibility of animation, basically it is possible to rotate curves, surfaces, change a static picture to other one by changing the parameter of solution. In GeoGebra animation is widely presented: rotations, changes of solution of the problem simultaneously with changes of the values of parameters by slider, geometrical changes by using of cursor and so on. It gives the possibility to investigate the behaviour of solution more detailed and helps in explanations.

On the base of this comparative analysis we suggest to use GeoGebra for visualization in presentations and explanations for students. The possibilities of Geogebra are wide, but usage of them requires some specific skills. MTV could be presented for students, who, as unexperienced users, can use it for control themselves by doing home works or repeating materials, for visualization a process of approximation of solution.

\section{Queueing systems}

One of the topics of Operations Research is the Queueing Theory. The problems of determination of optimum number of workers, flow of scheduled traffic, problems of loading and unloading, repairs and maintenance of equipment, etc. usually are considered by models of the queueing theory.

There are different special modelling and simulation tools for queueing problems. The authors of the paper in own practice deal with such software as JMT (Java Modelling Tools), CPN (Coloured Petri Nets) Tools, AnyLogic, Microsoft Excel QTP (Queueing ToolPak). In this paper only Java Modelling Tools (JMT) is considered in details as more prevalent, easy to use and free software. JMT is a suite of applications developed by the Politecnico di Milano and the Imperial College London and released under GPL license ([11]). The project aims are at offering a comprehensive framework for performance evaluation, system modeling with analytical and simulation techniques, capacity planning and workload characterization studies. The suite implements several state-of-the-art algorithms for exact, approximate, asymptotic and simulative analysis of queueing network models. Models can be described either through wizard dialogs or with a graphical user-friendly interface. The workload analysis tool is based on clustering techniques. For more detailed description of application JSIMGraph and the examples of using it see, e.g. [1]. After the definition of all necessary parameters we could obtain the results of simulations as the mean values and as confidence intervals (see Fig. 5). JMT gives also the possibility to conduct the analysis of changes of the performance measures depending of the values of some other parameter of the system What-if-Analysis.

It should be mentioned that the other application of JMT, Markov chain simulator (JCMH), could be used also for visualization of the concept of probability, namely the statistical definition of probability. 


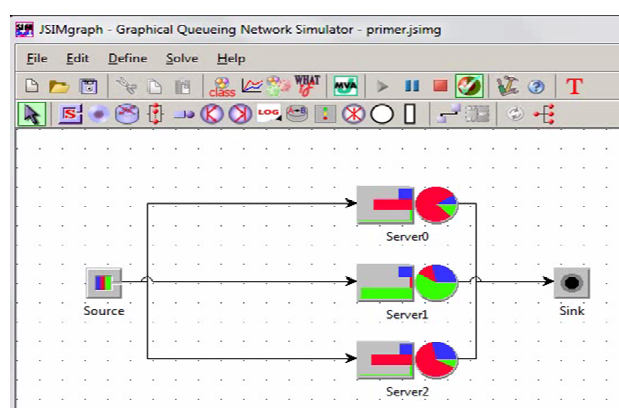

a)

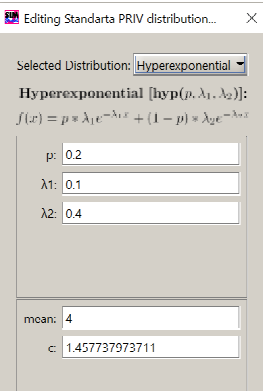

b)

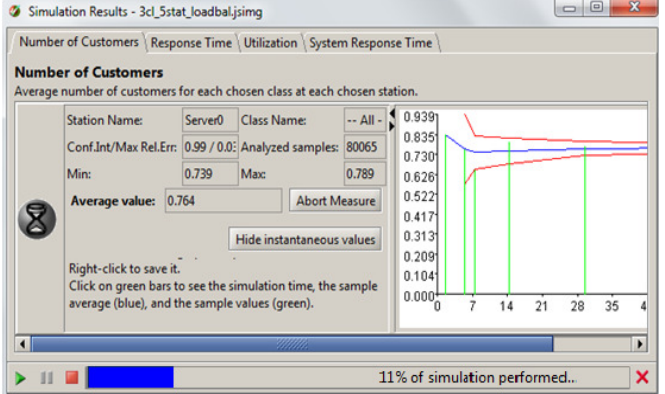

c)

Fig. 5. Graphical representation of model in JSIMgraph (a), definition of distribution (b) and results of simulations (c)

\section{Network scheduling}

The examples from another area of operations research - network scheduling - also could be presented as the examples for visualization. For this topic the authors recommend to use Microsoft Project (MS Project). MS Project is a project management software product developed and sold by Microsoft. The first commercial version was released for DOS in 1984. Now there are 20 million + users of this software package. It is designed to assist a project manager in developing a plan, assigning resources to tasks, tracking progress, managing the budget, analyzing workloads. The different activities and their relationships of the entire project are represented diagrammatically with the help of networks and arrows, which are used for identifying critical activities and path. Some notations from scheduling are repeated below: creating a schedule, scheduling methods and charts. The process of creating a schedule is deciding how to order the tasks and how to commit resources between the variety of possible tasks. A schedule or a timetable, as a basic time-management tool, consists of a list of times at which possible tasks are intended to take place. Scheduling methods are methods of arranging, controlling and optimizing work and workloads, obtaining the way in which various processes are assigned to resources that complete these processes. Scheduling aims to maximize the efficiency of the processes, to reduce costs and to minimize the time.
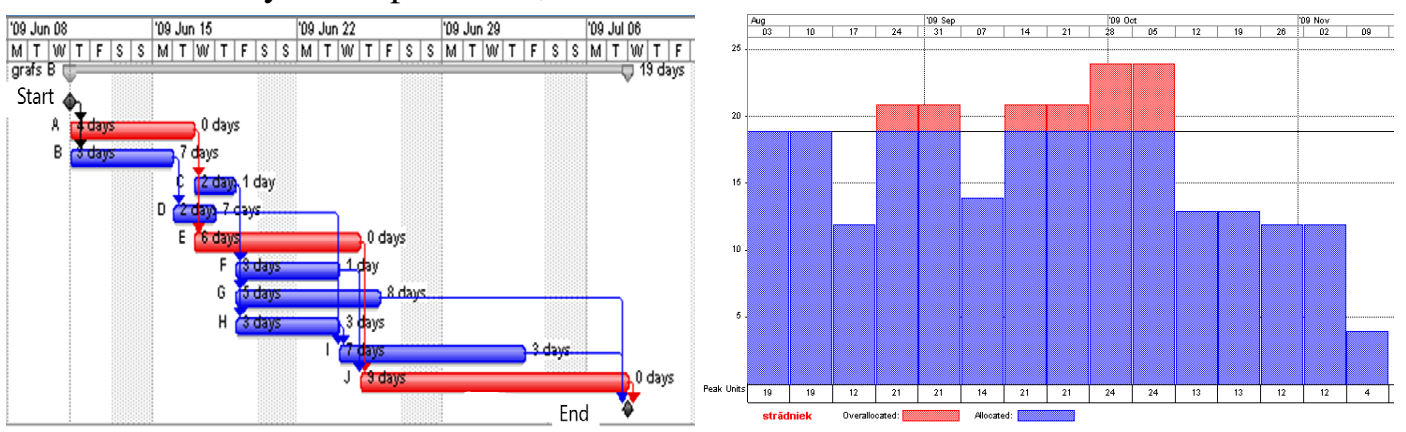

Fig. 6. Gantt chart and resource graph in MS Project

Various charts are used to help visually manage schedules and constraints. Figure 6 contains the Gantt chart - a display that shows activities on a graph, in which the time of each activity is represented. Recourse graph shows the time during which key resources are occupied (overallocated resources are showed by red color).

\section{Mathematical Statistics}

The examples from Mathematical statistics usually could be illustrated by diagrams, graphs, and so on. Usually these images are static. For construction of them different software could be used.

Data Plotter Application from Mathematical Visualization Toolkit allows to plot data points in 2$\mathrm{D}$ or 3-D and then connect the points to find trends in the data set, or approximate the data with a least square, linear fit to the data.

GeoGebra allows to visualize and analyse many concepts from statistics: analysis of the sample (mean, median, range and so on), analysis of dependence between the sample size and variance, 
analysis of the changes of the continuous random variable distribution function in dependence on parameters; regression analysis, etc.

RStudio is more effective software for statistics, which allows not only obtain the statistical data analysis, but also visualize the obtained results. RStudio is professional software for the R statistical computing environment ( $\mathrm{R}$ is free flexible and powerful foundation for statistical computing).

RStudio has four windows, as shown in Figure 7: Code editor is for creating the file containing $\mathrm{R}$ script, $\mathrm{R}$ console is for typing $\mathrm{R}$ commands, $\mathrm{R}$ environment and history is for viewing command histories and Graphical output is for viewing plots and more. The menus have all the options found in the standard R GUI, which are more useful for those who wish to write and debug their own R functions and packages. But also for non-programmers the tight integration of functions (console window) with a viewer for output charts and help information is very convenient. The mission to provide the most widely used open source and enterprise-ready [12].

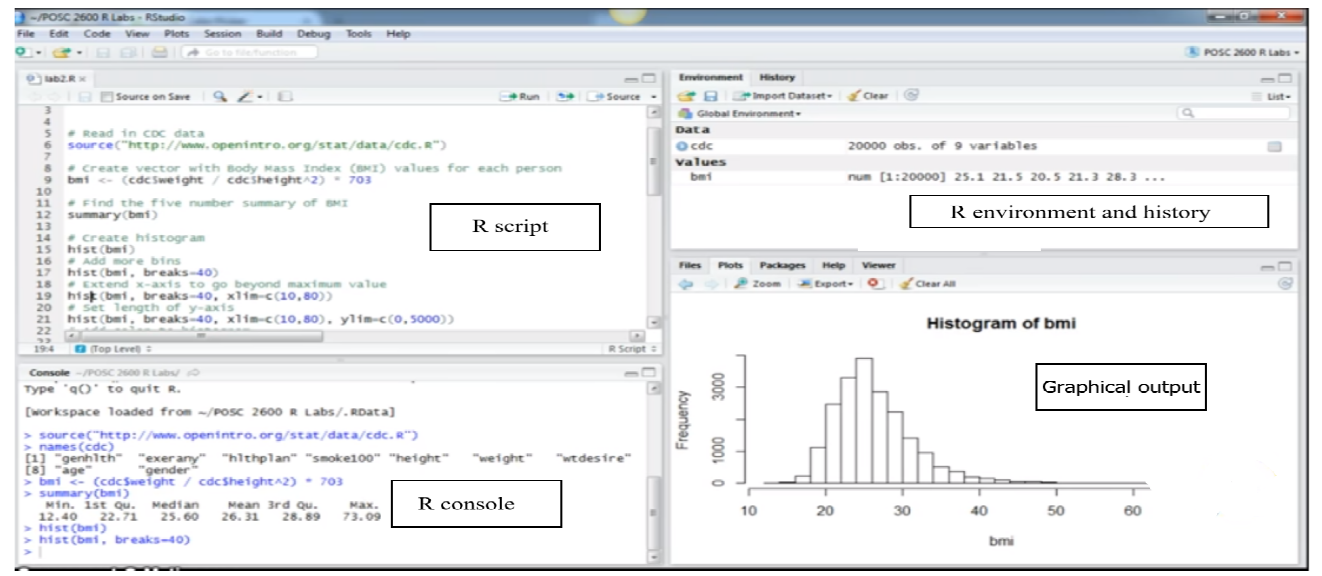

Fig. 7. RStudio windows

RStudio is actively used by students of the professional programme Mathematics Statistics at the University of Latvia for the solution of different problems of statistics.

\section{Visualization software in Bachelor's and Master's Theses}

The possibilities of effective using of the visualization technique by software considered in the previous paragraph for modelling and analysing of real-world systems allow apply it when working out study projects, bachelor's and master's theses. Let us show some examples of bachelor's and master's theses defended at the University of Latvia and the Riga Technical University, where such possibilities are used.

The most of these theses are devoted to the queueing systems. For example, Laura Birzniece "Application of Queuing Theory for Resources Forecast" (2017), Alīna Grīnberga "Modelling of Queueing Systems using CPN Tools" (2015), Aiva Baumane "Application of Queuing Theory in Analyzing the Company's Performance" (2014), Liene Ziedina "Analysis of Call Centre Performance with Simulation Method" (2013), Dace Bernāne "Information Service as a Model of the Queuing Theory System" (2013), Rimma Golovenko "Analysis of Work of the Bank Customer Service Centre" (2012), Jel̦ena Jakovlıeva "Functional Analysis of Store" (2012). The base of analysis in these theses is such software packages as JMT and CPN.

The other field of the defended theses is network scheduling. For example, Līga Kokoreviča "On Two Optimization Problems in Planning of Projects" (2011), Vita Tilgase "Project Planning and Realization Time Optimization Using "MS Project" program" (2009). Bachelor's thesis "Binary Programming Methods for Scheduling Optimization Problems" (2016) of Līga Koškina is also devoted to network scheduling, as well as PhD thesis of Ansis Ozolinšs "Shop Scheduling Problems: a Dynamic Programming Approach" worked out at the University of Latvia (will be defended in 2019).

\section{Conclusions}

The paper is written to share the authors' experience in using visualization and simulation techniques in teaching Mathematical Analysis; Methods of Optimization; Operations Research; 
Probability Theory and Mathematical Statistics. The authors deal with such special tools as GeoGebra, MVT, JMT, RStudio. The considered examples show how the considered tools could be effectively used for different aims of the study process:

- for explanation and illustration of theoretical results (during lectures and practical works);

- for analysis of real-world systems (working out study projects or bachelor's theses);

- for research in the corresponding fields of mathematics (working out master's or doctoral theses).

The authors' experience of using visualization show the importance of it in teaching mathematics and prove the necessary to use it in the teaching practise in future.

\section{References}

[1] Arcavi A. The role of visual representations in the learning of mathematics. Educational Studies in Mathematics, 52, 2003, pp.215-241.

[2] Asmuss S., Budkina N. Adaptation of course of operations research to needs of engineering study programmes by including specific models and example. Engineering for Rural Development, V. 17, 2018, pp. 1236-1244.

[3] Bishop A. Review of research in visualization in mathematics education. Focus on Learning Problems in Mathematics, 11(1), 1989, pp. 7-16.

[4] Presmeg N. Visualization and Learning in Mathematics Education. In: Lerman S. (eds) Encyclopedia of Mathematics Education. Springer, Dordrecht, 2014.

[5] Presmeg N. Research on Visualization in Learning and Teaching Mathematics. In A. Gutiérrez, \& P. Boero (Eds.), Handbook of Research on the Psychology of Mathematics Education: Past, Present and Future. Rotterdam: Sense. 2006, pp. 205-236.

[6] Zimmermann W., Cunningham S. Editor's introduction: What is mathematical visualization. In W. Zimmermann \& S. Cunningham (Eds.). Visualization in Teaching and Learning Mathematics. Mathematical Association of America, Washington, DC., 1991, pp. 1-8.

[7] Hoyles C., Lagrange J.-B. Mathematics Education and Technology - Rethinking the Terrain: The 17th ICMI Study. Springer, 2009.

[8] Souto V.T. Interactive Visualizations in Learning Mathematics: Implications for Information Design and User Experience. In: Marcus A. (eds) Design, User Experience, and Usability. User Experience Design for Diverse Interaction Platforms and Environments. DUXU 2014. Lecture Notes in Computer Science: Springer, Cham, vol. 8518, 2014.

[9] Introduction to GeoGebra. Version 4.4. [online] [15.03.2019]. Available at: http://static.geogebra.org/book/intro-en.pdf.

[10] Mathematical Visualization Toolkit [online] [15.03.2019]. Available at: http://amath.colorado.edu/java/history.php.

[11] Performance Evaluation Modelling with JMT: Learning by Examples, G. Serazzi Ed., Politecnico di Milano, DEI, TR 2008.09, 2008.

[12] RStudio [online] [15.03.2019]. Available at: https://www.rstudio.com/. 